\title{
Preparation and application of molecularly imprinted polymers for chiral HPLC separation of biologically active substances
}

\author{
Anna Lomenova ${ }^{\bowtie}$ and Katarína Hroboňová ${ }^{\bowtie}$ \\ Institute of Analytical Chemistry, Faculty of Chemical and Food Technology, Slovak University of Technology in Bratislava, \\ Radlinského 9, Bratislava SK-812 37, Slovak Republic
}

\section{Article info}

\section{Article history:}

Received: $17^{\text {th }}$ January 2020

Accepted: $6^{\text {th }}$ March 2020

\section{Keywords:}

Amino acids

Enantiomers

HPLC

Chiral stationary phase

Molecularly imprinted polymers

\begin{abstract}
Chiral separations are one of the important analytical tasks, since there are increasing demands for production of enantiomerically pure compounds. The separation and determination of enantiomers find applications in pharmaceutical and food analysis, and it is necessary to pay attention to the development and improvement of chiral analytical methods. High performance liquid chromatography (HPLC) with chiral stationary phase (CSP) based on molecularly imprinted polymers (MIPs) is perspective way. One of the main advantage of these stationary phases is the possibility of predetermining the elution order of enantiomers. The presented work is focused on the methods of preparation and the applications of selective sorption materials (MIPs) in the field of HPLC separation of biologically active substances, amino acids. This review contains comprehensive informations about MIP-amino acid synthesis: compositions of polymerization mixture (monomer, template, cross-linker, porogen), type of polymerization and polymerization conditions, what can affect final efficiency of enantioseparation. The most used porogen was toluene, crosslinker ethylene glycol dimethacrylate (EDMA) and initiator azoisobutyronitrile (AIBN). MIP CSP prepared for derivatized amino acids show better results (higher resolution) than MIP prepared for underivatized amino acids. MIPs are very promising material to be used as stationary phase in HPLC, although further developments and new approaches are necessary to fully exploit their potential.
\end{abstract}

(C) University of SS. Cyril and Methodius in Trnava

\section{Introduction}

Technology of molecular imprinting is very exploited technique in the field of separation science to obtain highly selective polymer material for analyte, or structurally related analytes, which are used in process of polymerization. Molecularly imprinted polymers (MIP) are prepared by reaction/interaction of analyte (template; for chiral applications the template is enantiomer) and functional monomer in presence of crosslinking monomer, initiator of polymerization and porogen. In first step, the functional monomer forms a complex with template molecule, which is subsequently polymerised in the presence of crosslinker and initiator to form stabile threedimensional polymer network. The template removal from polymer network reveals cavities complementary to the size, shape and chemical functionality arrangement to those of the template. Specific binding sites allows selective rebinding of the analyte from complex matrices (Fig. 1) (Cheong et al. 2012).

When synthesis of MIP takes place, it is very important to select an optimal template molecule, because it affects the process of MIP recognition 


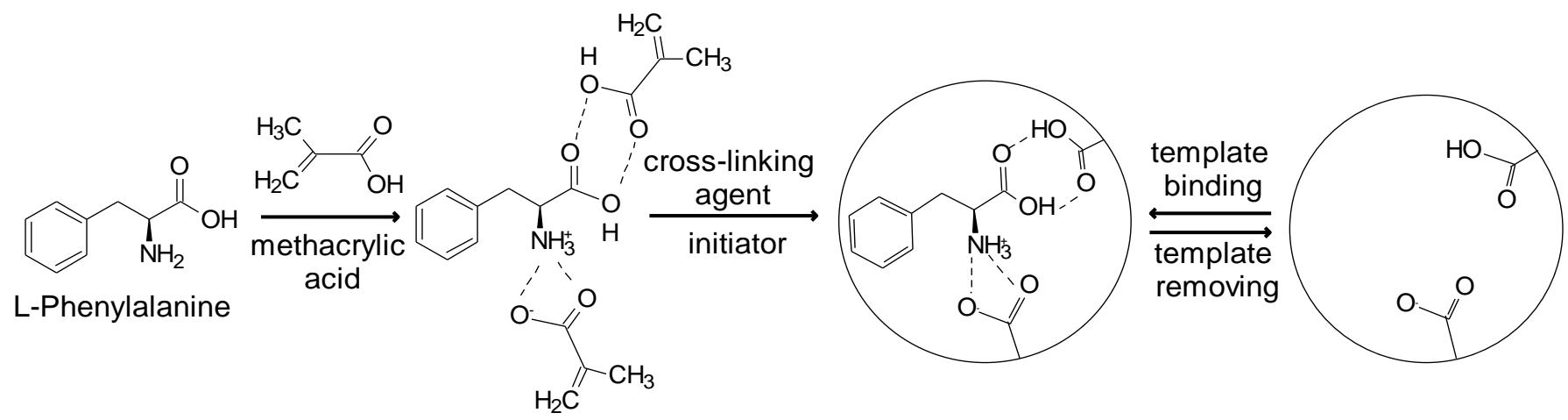

Fig. 1. Example of MIP preparation for template L-phenylalanine and predicted types of interactions with monomer methacrylic acid in imprinting process.

ability. The ideal template should have good chemical stability during polymerization process and it should have functional groups that can form complex with monomer (Chen et al. 2016). MIP can also be prepared for so called ,dummy" template (molecule similar to target analyte in terms of shape, size and functionalities). The main advantage of "dummy" templates is that have no interferences in analytical determination of target analyte. These approach is chosen when the template is too toxic to handle, not available in sufficient amount, expensive, or to avoid template-bleeding problem when MIPs are used for trace analysis (Song et al. 2017).

Monomer in polymerization mixture affects the formation of highly specific cavities for template. It must contain functional groups that strongly interact with functional groups of template molecule to give stabile complex. Basic monomers (e.g. 2-vinylpiridine (2-VP) (Li et al. 2017), 4-vinylpyridine (4-VP) (Kibechu et al. 2017), $N$-vinylimidazole (VIm) (Llorina Rañada et al. 2014) or acidic monomers (e.g. methacrylic acid (Hroboňová and Lomenova 2018), acrylic acid (AA) (Liu et al. 2013), itaconic acid (IA) (Gutiérrez-Climente et al. 2016) were used in many applications. Among all monomers, methacrylic acid (MAA) is universal and the most used monomer due its functional groups which can act like hydrogen donor (hydroxy group) and acceptor (carbonyl group) (Fig. 1).

The crosslinking agent during polymerization fixes the functional groups of the monomer around the template molecule to form a solid cross-linked polymer. In addition to stabilizing the resulting binding sites, the type and amount of cross-linking agent also affects the morphology and recognition ability of MIP. Very low amount of cross-linker leads to formation of polymer with unstable mechanical properties due to the low cross-linking degree, and on the other hand, very high amount of cross-linker can reduce the number of recognition sites per unit mass of MIPs. An optimum percentage of crosslinker is between $50 \%$ and $80 \%$ from mass of used monomer (Spivak 2005). Most commonly used are ethylene glycol dimethacrylate (EDMA) (Hroboňová and Lomenova 2018), trimethylolpropane trimethacrylate (TRIM) (Olcer et al. 2017), divinylbenzene (DVB) (Nakamura et al. 2017) and other.

In the synthesis of polymers, attention is also paid to selection of type and volume of the solvent used. The solvent dissolves the polymerization components and is responsible for pore formation. It should be to ensure the formation of pores to allow good flow of mobile phase through the polymer. Solvents, such as toluene, chloroform, dichloromethane, or acetonitrile may be used. The choice of solvent type also affects the formation of the complex template-monomer, and finally the adsorption properties of MIPs. Less polar solvents increase the possibility of forming a stable complex by facilitating the formation of polar non-covalent interactions (hydrogen bonds), while more polar solvents interfere creation of hydrogen bonds in the template-monomer complex (Chen et al. 2016).

Many types of initiators can be used for polymerization, which differ in chemical properties and serve as a source of radicals in radical polymerization. Radical formation can be initiated 
either thermally or photochemically depending on the kind of initiator used. Initiator is added to the polymerization mixture in a small amount compared to the monomers (approximately $1-10 \%$ from amount of monomer). The most common used initiators are azo-compounds (e.g. azoisobutylonitrile (AIBN) (Hroboňová and Lomenova 2018), 2,2'-azo-bis(2,4-dimethyl valeronitrile) (ABDV) (Kibechu et al. 2017), 4,40-azo(4-cyanovaleric acid) (ACID) (Chen et al. 2016).

Depending of types of interactions/bonds between analyte and functional monomer in prepolymerization complex, MIP can be prepared by covalent and noncovalent approach. Covalent approach is based on formation of covalent, irreversible bonds between template and monomer. For the template removal, chemical cleavage of the supporting covalent bonds was used. Cavities are highly compatible with molecules of analyte, but kinetics of the binding and rebinding processes may be slow. Another way for MIP preparation is noncovalent approach, which utilize noncovalent interactions (e.g. hydrogen bonds, Van der Waals and electrostatic interactions) between monomer and template during both, the imprinting procedure and the rebinding. Because of weaker reversible interactions, template can be removed from the imprinted sites by extraction with organic solvents or aqueous solution of an acid or base. In imprinting procedure also semi-covalent approach is used, which is combination of covalent and noncovalent approach. Covalent bonds between template and monomer are formed during the polymerization and during the rebinding of analyte from solution, only noncovalent interactions are used. In practice noncovalent approach is the most used, because of more flexible choice of functional monomers and possible target molecules (Yan and Row 2006; Maier and Lindner 2007).

Beside high affinity and target specificity, MIPs possess advantages such as high physical and chemical robustness (resistance of high pressure and temperature, organic solvents, acids and bases), ease preparation, low-cost production and reuse. On the other hand, MIPs have also disadvantages. Time consuming optimization of conditions for their preparation, which include selection of compounds of polymerization mixture (monomer, crosslinking agent and porogen), their ratio, as well as reaction conditions (temperature and time of polymerization) to form polymer with required properties (high sorption capacity, selectivity, morphology) (Cheong et al. 2012). Due to their properties, MIPs have great potential in analytical applications, in biosensors (Nguy et al. 2017; Selvolini and Marrazza 2017), capillary electrophoresis (Alenazi et al. 2015; Li et al. 2017; Giovannoli et al. 2018), high performance liquid chromatography (Ndunda and Mizaikoff 2016; Yang et al. 2018), supercritical fluid chromatography (Ansell et al. 2012), or even in sample treatment procedures such as solid phase extraction (Bujak et al. 2016; Lucci et al. 2017), solid phase microextraction (Szultka et al. 2013; Turiel et al. 2016) and stir bar sorptive extraction (Fan et al. 2016; Tang et al. 2018).

\section{Preparation of MIP as stationary phases for HPLC}

Molecularly imprinted polymers are used in HPLC mostly for enantioseparation. Comparing to commercially available chiral HPLC column which are used for separation of enantiomers in daily analyses, molecularly imprinted polymers (MIPs) are sorbents with a higher, predetermined selectivity for a given enantiomer. Using of these materials as a chiral stationary phases (CSP) is particularly limited due to the difficulties associated with the formation of particles suitable for column filling. Therefore, the development of polymerization processes for obtaining MIP particles with the desired parameters (shape, size) is of great importance.

\section{Bulk polymerization}

The molecularly imprinted polymers are most often prepared by bulk polymerization. Polymerization mixture consists from template, functional monomer, crosslinking agent, initiator of polymerization and porogen. Polymers are formed through free radical polymerization, which can be thermally or photo initiated. Final, solid polymeric block must be crushed and sieved to obtain particles with desired size (smaller than 


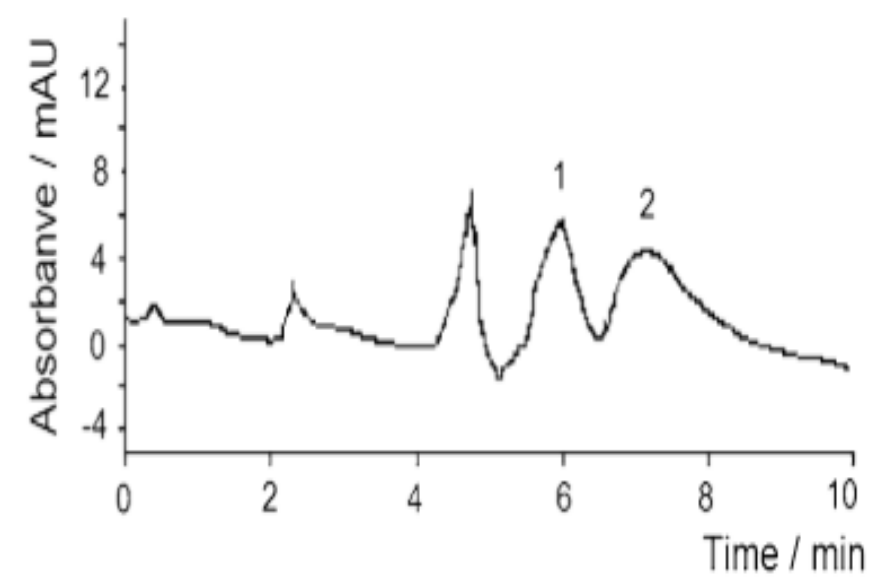

Fig. 2. Separation of phenylalanine enantiomers on MIP-Lphenylalanine stationary phase prepared by bulk polymerization (Hroboňová and Lomenova 2018).

$25 \mu \mathrm{m})$. Template is removed from cavities by extraction and polymer is packed into chromatographic column. Although this type of polymerization is simple, such a polymer preparation is time consuming, obtained particles have heterogenous shape and size and crushing the polymeric block can also damage the formed cavities. Particles prepared by bulk polymerization are less suitable because low mass transfer kinetics are often observed, what have impact on enantioseparation (Fig. 2) (Vasapollo et al. 2011; Zheng et al. 2011). In order to obtain particles with desired characteristic, what lead to improved chromatographic characteristics, different types of polymerization techniques, such as precipitation, suspension, multistep swelling, polymerization in preformed beads and surface imprinting, have been used.

\section{Precipitation polymerization}

Compared to block polymerization, precipitation polymerization occurs in the presence of a larger amount of porogen (10-times higher than in bulk polymerization) that dissolves the monomer (usually monomer concentration is $<5 \%$ of total mass), template, crosslinking agent, and initiator, but does not dissolve the resulting polymer. The polymer particles tend to accumulate and precipitate from solution to form micro-gel particles (Pardeshi and Singh 2016). It is one of the most convenient and easiest procedures (surfactant free, no need of wasteful and time-consuming procedures) to form MIP particles with desired particles (particles size $3-5 \mu \mathrm{m}$, homogenous distribution of binding sites) and high yield. Porosity and size of particles can be controlled through polymerization conditions. It was found that the most important is matching the solubility parameter of developing polymer to solubility parameter of porogenic solvent (Turiel and MartinEsteban 2004; Yoshimatsu et al. 2007).

\section{Suspension polymerization}

For MIP preparation by suspension polymerization two-phase system is used. The monomer dissolved in the organic solvent is mixed in an excess of water containing the suspension stabilizer (e.g. polyvinyl alcohol). During mixing, droplets of organic phase with homogenous size and shape are formed in the aqueous phase. This type of suspension polymerization is rarely used because water can limit the formation of hydrogen interactions between template and monomer. It is used when electrostatic and hydrophobic interactions are strong enough (Turiel and MartinEsteban 2004). Better alternative to conventional suspension polymerization is polymerization where perfluoroalkane solvents are used instead of water. These low polarity solvents are able to stabilize noncovalent interactions, especially hydrogen bonds, between template and monomer. In this way, particles with size from 5 to $50 \mu \mathrm{m}$ are formed, depending on the amount of stabilizer used (Maier and Lindner 2007).

\section{The imprinting in preformed beads}

The imprinting in preformed beads method is based on polymerization in the cavity of a porous carrier (e.g. silica gel). First, the pores of the carrier are filled with the polymerization mixture, they are heated while polymerization takes place and a composite material (silica gel-MIP) is formed. The silica gel can be dissolved and removed leaving spherical particles consisting only of MIP (Yilmaz et al. 2002). Spherical MIP exhibits less back pressure, higher mass transfer and a higher number of theoretical plates compared to conventional block polymerization particles. Nevertheless, peak tailing can be monitored. To increase the mass transfer, it has been proposed to anchor the template in the carrier cavities. 
After polymerization and dissolution of the silica gel, complementary pores containing binding sites were obtained which are located on the surface of the resulting polymer (Turiel and MartinEsteban 2004).

\section{Multi-step swelling polymerization}

The multi-step swelling polymerization is best polymerization for the synthesis of monodisperse particles in high yield. It uses polystyrene latex beads in water with added stabilizer. A low molecular weight activation solvent (e.g. dibutyl phthalate (Haginaka and Kagawa 2004) and initiator of polymerization are added to the solution. Under the stirring or ultrasonication, the components are absorbed into the latex beads, causing swelling. The swollen beads are added to the mixture containing the monomer, template, crosslinking agent and solvent in an aqueous medium in the presence of polyvinyl alcohol as a stabilizer. The solution is stirred until the dispersion droplets of the polymerization components are absorbed into the latex beads. After polymerization, MIP particles with spherical shape and small distribution in size $(5-10 \mu \mathrm{m})$ are formed. Such a polymer is resistant to high temperatures and pressures, which allows using it at higher flow rates (Turiel and Martin-Esteban 2004).

\section{Surface polymerization}

Another possibility is to synthesize MIP on the surface of the carrier (e.g. silica particles (Gutiérrez-Climente et al. 2016), chitosan (Wang et al. 2009) and activated polystyrene particles (Qin et al. 2009) with defined shape and size. For polymerization can be applied "grafting to" or "grafting from" approach. In most used "grafting to" polymerization, the carrier is modified with double bonds on the surface and in presence of the imprinting mixture the free-radical polymerization will start. This lead to copolymerization of monomers and double bonds on the surface and at the end to formation of MIP coating. With this approach, stabile polymeric film can be prepared. Since the initiator is in the solution, polymerization doesn't take place only at the surface of the carrier, but polymers are also created in solution. That leads to harder control of the film thickness. To improve these procedures, "grafting from" approach is used. Initiating radicals are attached directly onto surface of carrier and polymer chain propagation will take place only on the surface and polymerization in solution is minimalised. An initiator transfer agent terminator (iniferter) is used. It is a compound (cumyl dithiobenzoate (Li et al. 2015), 4-cyano-4-(phenylcarbonothioylthio) pentanoic acid (Li et al. 2014), benzyl dithiocarbamate (Abdollahi et al. 2016) that acts as radical initiator, chain transfer agent and polymerization terminator. This kind of initiator decomposes into two radicals, active and dormant. Active radical is located on the surface of the carrier and initiates polymerization while the other is stable, in solution, and prevents unwanted polymerization. In this process density and thickness of the resulting polymer layer can be better controlled (Tan and Tong 2007; GutiérrezClimente et al. 2016).

\section{In-situ polymerization}

In situ polymerization is based on monoliths that are formed by radical polymerization directly in the HPLC column (compared to the processes where at first the MIPs are synthetized and then columns are filled with obtained sorbent). This process combines the advantages of monolithic columns (e.g. mechanical stability due one-piece structure, high permeability based on large porosity and large number of theoretical plates, which is related to high efficiency and highspeed separations and molecular imprinting technology (Tanaka and Kobayashi 2003). The column is filled with the polymerization mixture, followed by heating or UV radiation to form a porous polymer. After polymerization, the template and solvent are washed with a suitable extraction solvent. Such stationary phases are characterized by ease of preparation, reproducibility and rapid mass transport. In polymerization, very important and limiting factor is the choice of porogen, which provides sufficient porosity of the monolith. This allows a good mobile phase flow through the column with 
low back pressure at higher flow rates. The greatest porosity is achieved by polar solvents that can affect the interactions between the monomer and the template, affecting the quality of the binding sites (Zheng et al. 2011).

\section{Efficiency of separation using MIP chiral stationary phases}

As was mentioned before, size and shape of MIP particles are very important for its application as HPLC stationary phase because they can affect peak shape and the efficiency of column. It has been found that using MIPs prepared by common polymerization techniques (bulk, suspension, precipitation, the imprinting in preformed beads or multi-step swelling polymerization), efficiency of separation is not sufficient. With these methods is difficult to create particles with desired properties (uniform shape, size, surface area) with high affinity of binding sites. That is caused by location of binding sites in polymeric particles. Part of binding sites are inside particles, while other are on the surface, which can lead to differences of analyte binding to polymer (adsorption and desorption) and thus to peak broadening. To improve kinetic of separation, surface polymerizations on different types of cores were proposed. Higher efficiency of separation is observed due to higher availability of binding sites in thin MIP layer. These stationary phases are characterised by high binding capacity and rapid sorption and desorption of analyte (Balamurugan et al. 2012; Lomenova and Hroboňová 2019).

Although, efficient enantioseparation can be reached by choosing sufficient polymerization components and polymerization technique, using MIP based CSP usually broad peaks with little tailing can be observed (Fig. 3). That can be caused by many reasons. One of them is different strength of interactions between high affinity binding sites of polymer and template molecule or its enantiomer, respectively. That lead to slower mass transfer rate when template takes place, and thus to peak tailing of template enantiomer. Heterogeneity of binding sites, non-specific interactions, high degree of crosslinking and mass overloading also play crucial role. When high amount of crosslinker or monomer are used during

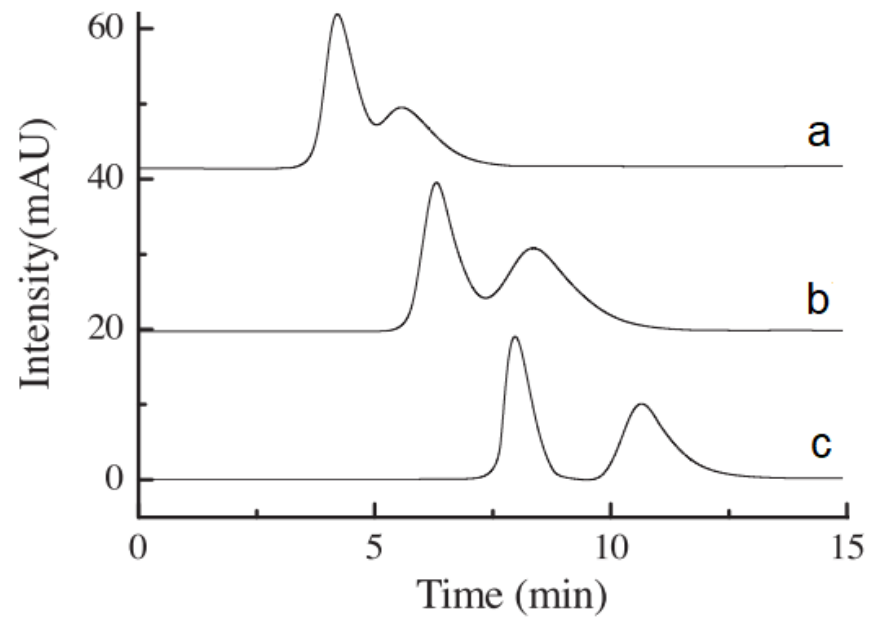

Fig. 3. Separation of phenylalanine enantiomers by MIP-Lphenylalanine prepared by surface polymerization, effect of monomers on efficiency of separation (a) MAA, (b) Acryloyl- $\beta$-CD and (c) MAA, Acryloyl- $\beta$-CD (Zhang et al. 2012).

the polymerization process, beside high affinity binding sites, in polymeric structure are also present free functional groups, that can provide non-specific interactions with enantiomers. During application of solution with lower concentration of ananlyte, analytes will interact only with binding sites through specific interaction. Increasing of analyte concentration results to occupation of polymeric cavities and analytes start to interact with free functional groups to give non-specific interaction leading to different mass transfer and thus affecting the peak symmetry (Tamayo et al.2005; Lomenova and Hroboňová 2019).

\section{MIP as chiral stationary phases for enantioseparation of amino acids}

Amino acids are chiral compounds and their enantiomers act differently in human body. Content of amino acid enantiomers are highly controlled in pharmaceutical and food industry. Naturally preferable in foodstuff are L-amino acids, D-enantiomers occurrence can indicate unsuitable food storage or industrial process and microbial contamination. Enantiomeric ratio of amino acids can provide information of food origin. Last years, food supplements containing amino acids are very popular. They can contain only L-forms, because D-forms can have different biological of physiological properties and may not be metabolized efficiently, what can lead 
to nutritionally poorer and less safe products (Sánchez-Hernández et al. 2016). On the other hand, occurrence of some D-amino acids in human body can help diagnose some diseases, e.g. Alzheimer, which find application of enantiomeric separation also in medicine (Fujii et al. 2018; Lomenova and Hroboňová 2018).

One of the possibilities for separation and determination of amino acid enantiomers is direct HPLC separation by using stationary phases consist of chiral selectors with different functionalities. Indicated by number of published papers, this type of chiral separations are well researched and according to literature, the most popular chiral stationary phase (CSP) for enantioseparations of amino acids are based on macrocyclic antibiotics - teicoplanin (Kučerová et al. 2013; Taujenis et al. 2014; Min et al. 2015; Riesová et al. 2016; Hroboňová et al. 2017), teicoplanin aglycone (Bystrická et al. 2016; Hroboňová et al. 2015), ristocetin (Wagdy et al. 2014; Lomenova and Hroboňová 2018), vancomycin (Deáková et al. 2015); polysacharide based chiral stationary phases - cyclodextrins (Kučerová et al. 2016; Hroboňová et al. 2017), cyclofructans (Hroboňová et al. 2017), amylose (Kučerová et al. 2013; Riesová et al. 2016) and chiral ion exchangers (Reischl et al. 2011; Woiwode et al. 2018; Geibel et al. 2019.

Beside of commercially available CSPs, the innovative stationary phases to reach chiral separations are based on molecularly imprinted polymers. These sorbents are the most applied in field of affinity chromatography. Since they are prepared with high selectivity for one enantiomer, these materials are appropriate for enantioseparation and can be used as chiral stationary phases. Depend on enantiomeric form which is used as template during the polymerization, elution order of enantiomers can be determined. If L-enantiomer was used as template, this form will provide more interactions with functional groups of MIP and will elute with higher retention time (Gutiérrez-Climente et al. 2016; Hroboňová and Lomenova 2018). These highly selective polymers can be prepared also for molecules that contain more than one chiral centrum. For example, if polymer was prepared for dipeptide acetyl-L-phenylalanine-L-tryptophanmethyl ester (Ac-L-Phe-L-Trp-Ome), this peptide will be retained more strongly compared to other stereoisomers (LD, DL and DD) which provide weaker interactions with functional groups into cavities and because of that will elute in shorter retention times (Ramstriim et al. 1994).

Chiral stationary phases based on MIP for separation of amino acid enantiomers can be prepared with some of enantiomer of amino acids or their derivatives as templates. Selection of functional monomer depend on type of template (characteristic functional groups), which lead to formation of strong monomer-template complex. More frequent approach for MIP CSP preparation is use of derivatized amino acids as templates and also derivatives are enantioseparated. Presence of different functional groups in template results to multiple interactions with monomer and preparation of MIP with more specific binding sites. In this case, mostly used functional monomers are methacrylic acid, 4-vinylpyridine and 2-vinylpyridine and etylenglycol dimethacrylate as cross-linking agent. When amino acids esters or amides are used as templates (Phe- $\beta$-naphtylamide, Phe-anilide), methacylic acid seems appropriate for MIP synthesis. Strong ionpar interaction of amine or amide functional groups with - $\mathrm{COOH}$ functional group of monomer and hydrogen bonds of carbonyl or carboxyl functional groups are dominant during MIP formation (Sellergren and Shea 1993; Takeuchi and Haginaka 1999). Monomers, such as 2- and 4-vinylpyridine are selected when tert-butyloxycarbonyl- (Boc-), fluorenylmethyloxycarbonyl- (Fmoc-) or $\mathrm{N}$-carbobenzyloxy- (Cbz-) of amino acids as templates (e.g. tert-butyloxycarnonyl-L-3-nitrotyrozine (Boc3NT), fluorenylmethyloxycarbonyl-L-3-nitrotyrozine (Fmoc-3NT), or $N$-carbobenzyloxy-Ltryptophan (Cbz-L-Trp)) were used. Pre-polymerization complex monomer-template is formed ion-pair interactions through. In the case of Boc-, Fmoc- or Cbz- derivatives, methacylic acid is not suitable monomer, because of formation weaker hydrogen bonds in polar solvents occurred (Scorrano et al. 2011; Balamurugan et al. 2012).

Acrylamide as basic functional monomer is preferred for templates with acidic functional groups. Stronger hydrogen bonds cause formation of a less nonspecific binding sites. It may be attributed to the fact that the unbound acrylamide occurs as a dimer (Ansell 2005). 
It was also found out that monomers containing carboxylic groups provide hydrogen bonds with amino acids derivatives that are weaker in organic solvents. To prepare MIP with more specific binding sites and better recognition ability, mixture of two monomers could be used for MIP preparation (e.g. methacrylic acid and acrylamide) (Takeuchi et al. 1999).

HPLC separations of derivatives of amino acids enantiomers (Table 1) on MIP-CSPs were performed in reverse-phase (RP), normal phase (NP) and polar-organic separation mode (PO). Higher values of resolution were observed in $\mathrm{PO}$ separation mode $\left(R_{\mathrm{S}}=2.2\right)$ compared with NP separation mode $\left(R_{\mathrm{S}}=1.7\right)$ (MIP for template BocL-Trp prepared by bulk polymerization; separation of Boc-D,L-Trp).

Type of MIP polymerization also affected efficiency of HPLC enantioseparation, however the selection of separation conditions is significant for enantiorecognition on CSPs. Higher resolution was achieved with MIP prepared by multistep swelling polymerization $\left(R_{\mathrm{S}}=2.2\right)$ compared to MIP prepared in-situ polymerization $(R \mathrm{~S}=0.5$; NP separation mode) or by surface polymerization $(R \mathrm{~S}=1.7$; PO separation mode) (MIP for template Boc-L-Trp; separation of Boc-D L-Trp).

Comparing the results for polymers prepared by the same polymerization technique for different derivates of amino acids as templates, can be concluded that type of derivatization agent have no significant effect on resolution of enantiomers $\left(R_{\mathrm{S}}=0.7\right.$ for Cbz-DL-Trp and Fmoc-DL-Trp; HPLC conditions were identical).

Rarely, underivatized amino acids are used as templates during MIP chiral stationary phases preparation. Unlike derivatized, non-derivatized amino acids have less functional groups, which provide less interactions in MIP formation, that results in reduced MIP selectivity. The most used templates for MIP preparation are aromatic or heterocycle containing amino acids (phenylalanine, tyrosine, tryptophan) (Table 2). For polymerization, the most used functional monomer was methacrylic acid or its mixture with cyclodextrin. Acrylamide can be also used mostly in combination with modified $\beta$-cyclodextrin. Cyclodextrin (CD; cyclic oligosaccharides with hydrophilic surface and a hydrophobic cavity) provides the formation of multiple types of interactions with the template. Pre-polymerization complex is formed due to the hydrogen bonds on the surface of $\mathrm{CD}$, and also due to the inclusion of hydrophobic guest molecule inside the CD cavity (hydrophobic interactions). This results in higher resolution enantiomers in chromatographic separation (Qin et al. 2008).

Compared the $R \mathrm{~s}$ values for derivatized and underivatized amino acids enantioseparation on MIP-CSP prepared by same polymerization technique can be concluded that higher resolution was achieved on MIPs prepared for amino acids derivates as templates (Z-L-Phe-OH, $R_{\mathrm{S}}=2.4$; bulk polymerization) compared to those prepared for underivatized amino acids (D-Phe, $R_{\mathrm{S}}=1.0$; bulk polymerization).

Table 1 and 2 summarise polymerization conditions for MIP preparation and chromatographic conditions for chiral HPLC based on MIP stationary phase. Presented works are focused mostly on optimisation of MIP synthesis, what include choosing of suitable monomer, crosslinker, porogen and type of polymerization to obtain sorbent with suitable properties, chemical characterisation (binding capacity and adsorption isotherms) and morphological characterisation (FTIR spectrometry, Scanning Electron Microscopy, Transmission Electron Microscopy, Thermogravimerty) of prepared sorbents. In cited works, prepared polymers were tested only for separation of standards, where optimisation of chromatographic conditions was done (mobile phase, flow rate, column temperature).

In recent years, MIPs designed for amino acids find applications in sample pre-treatment like highly selective SPE sorbents. Recently they were used for medical and food analysis purposes: determination of glycine (Hashemi-Moghaddam et al. 2015) and 3-nitro-L-tyrosine (Mergola et al. 2013) in human urine, determination of amino acids from tobacco (Zhu et al. 2016) and determination of cysteine (Cai et al. 2014).

Nowadays, MIP have great application like stationary phases in pharmaceutical and food industry. All newly designed biologically active substances used in pharmacy are strictly monitored and need to fulfil regulations. Because of this, latest research if focusing to design polymers with high quality, what can help to achieve fast and effective enantioseparations. MIPs were designed for 


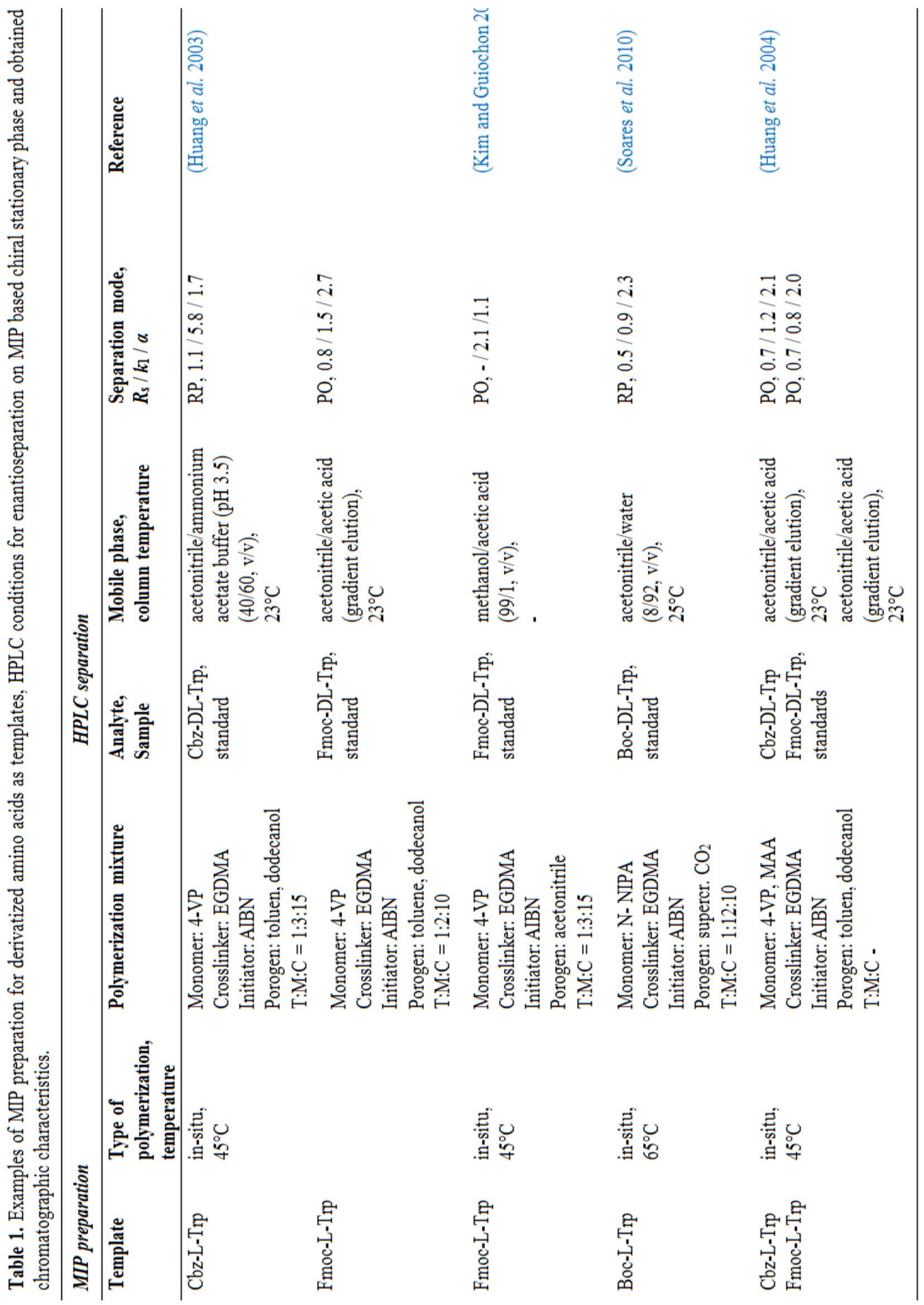




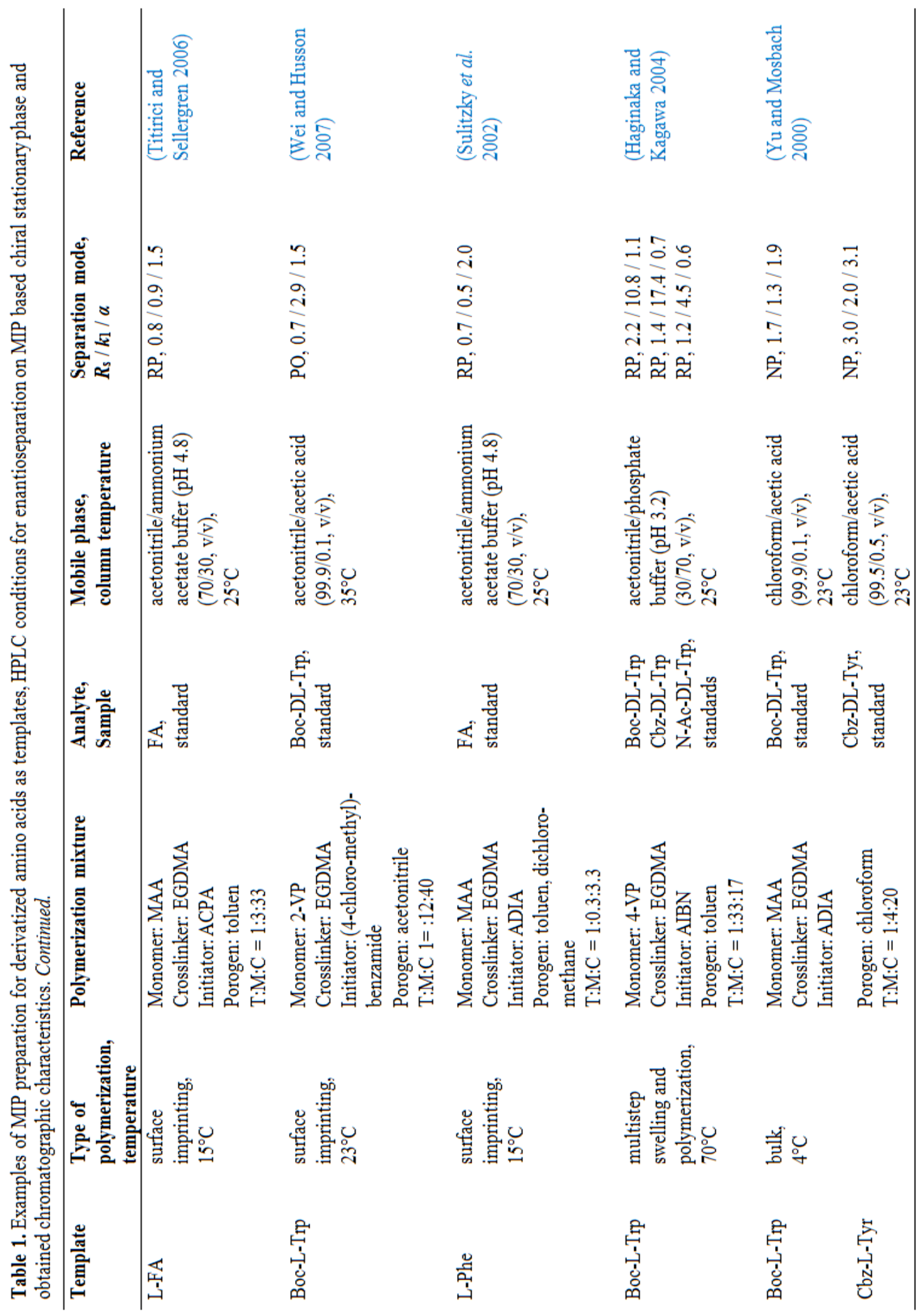




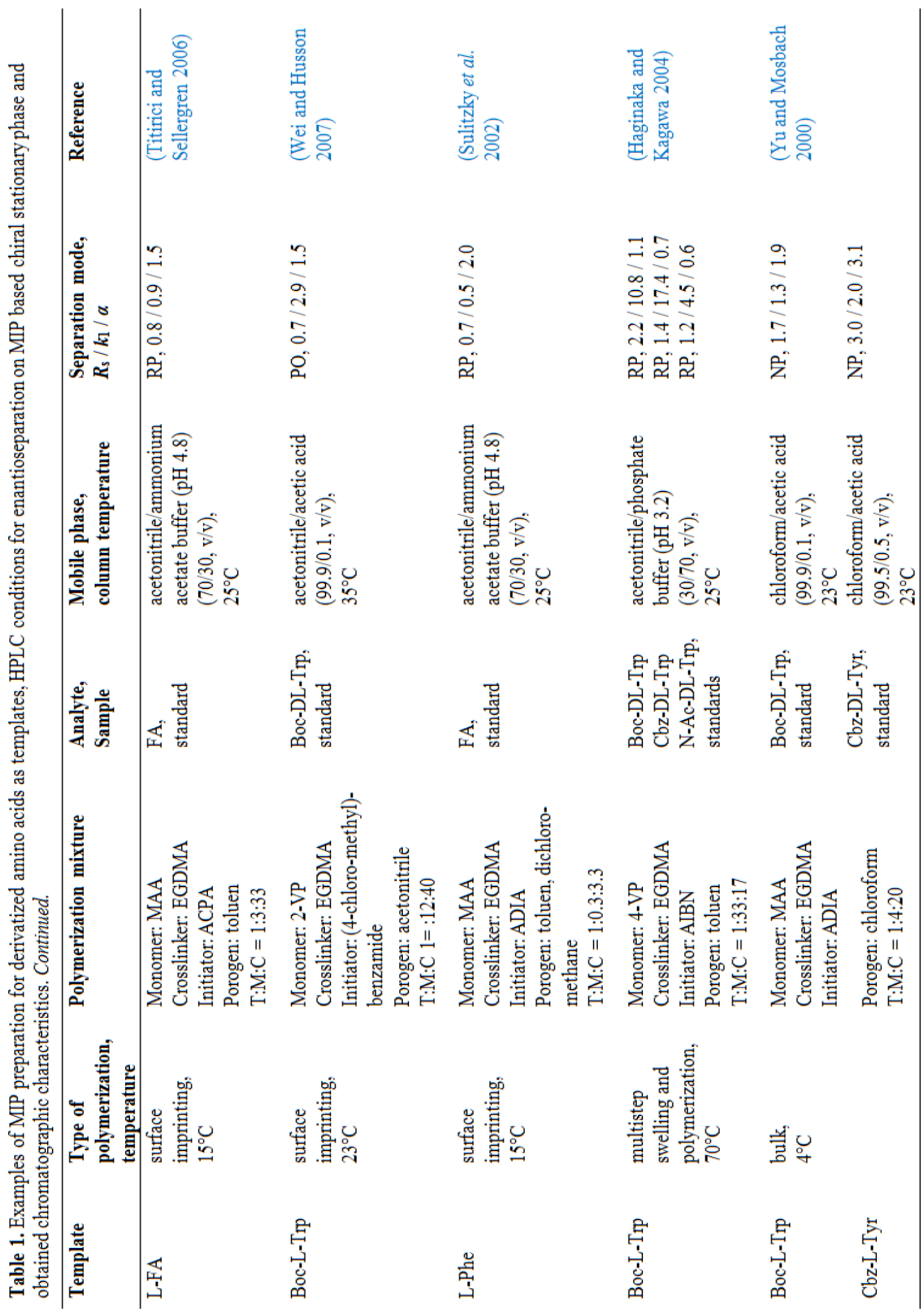




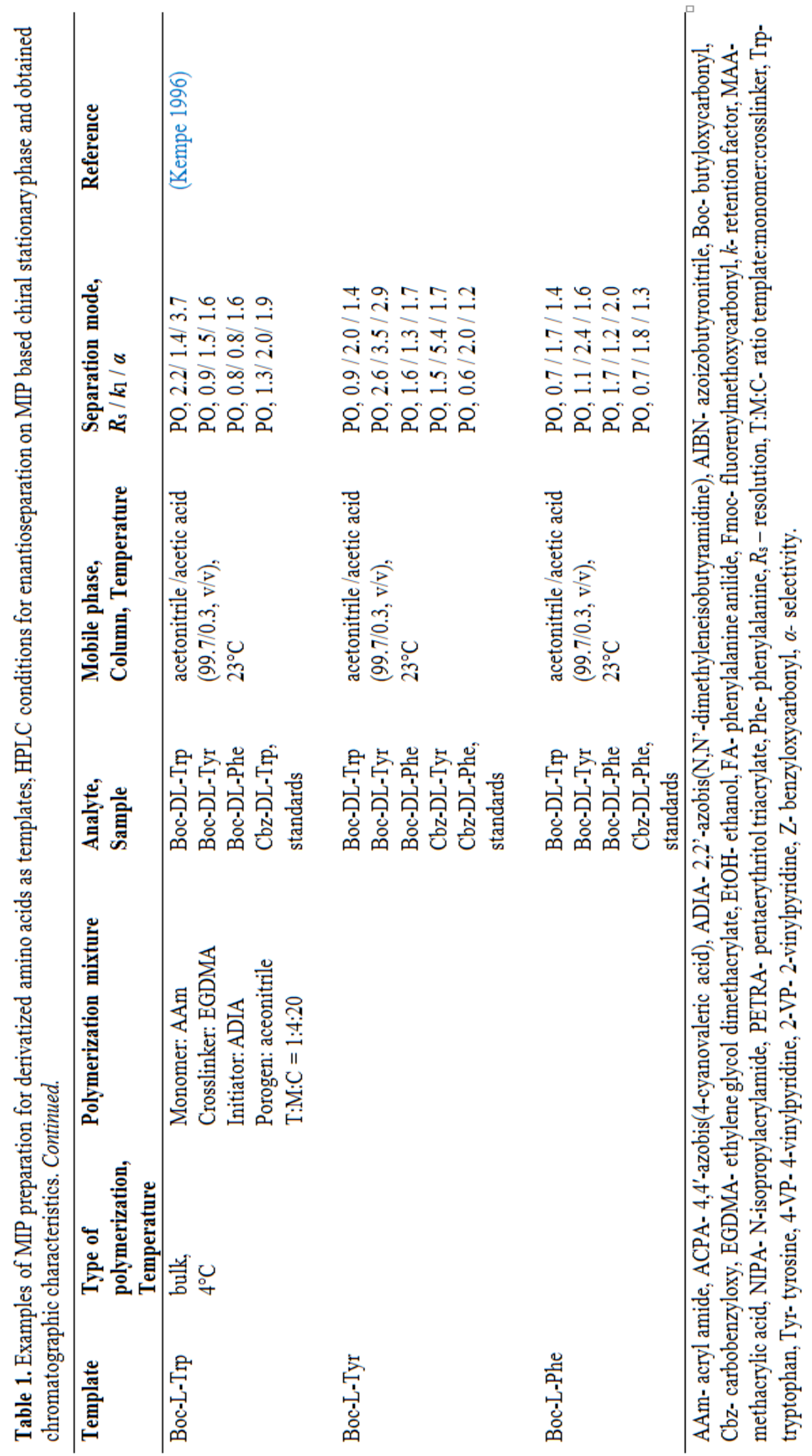




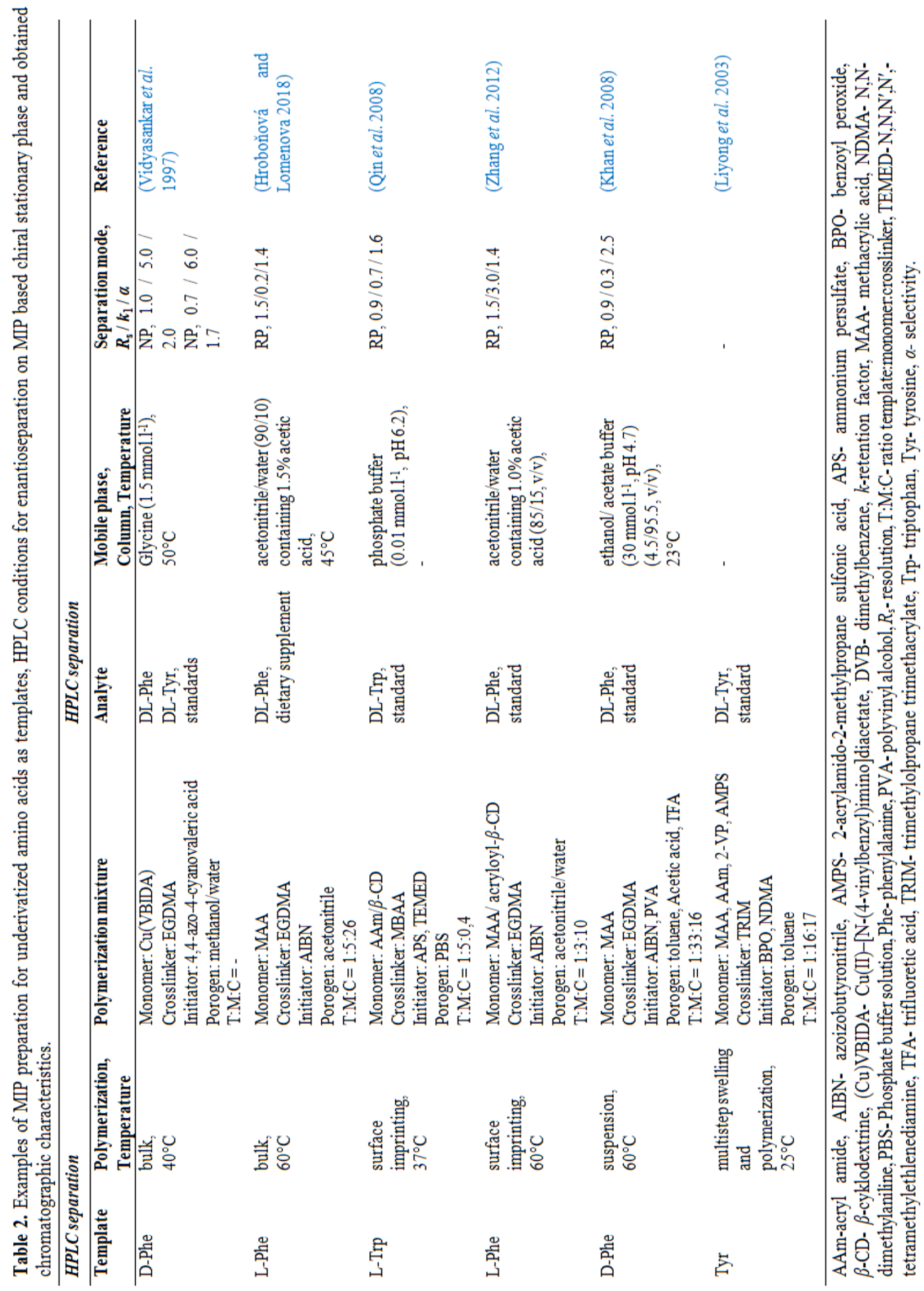


enantioseparation of medicament ephedrine (Ansell et al. 2012), citalopram (Gutiérrez-Climente et al. 2016), paclitaxel (Li et al. 2017) and cathine (Balamurugan et al. 2012). Another application find place in food chemistry, where determination of specific substances in products can give information about quality of foodstuff, for example 4-ethylphenol in wine (Garcia et al. 2015), or myricetin in plants (Xiao et al. 2016).

\section{Future Perspectives}

Although over the last years, synthesis of MIP reaches good progress, conventional polymerization techniques had some disadvantages. Many of them used large volumes of organic solvents which may lead to harder adaptation and application of MIPs in industry due to the due to the non-ecology approach. Use of water as solvent during polymer synthesis would be more environmental friendly alternative, but water can strongly interact with template/monomer a thus inhibit formation of hydrogen bonds (specific recognising interactions) between monomer and template. This can result in formation of less stable pre-polymerization complex and preparation of MIP with nonspecific binding sites.

To reduce the consumption of chemicals and its final release to the environment, the aim of research is the finding innovations in techniques of polymers preparation which will apply the principles of green chemistry. Great future potential have strategies using supercritical carbon dioxide (Da Silva et al. 2010) or ionic liquids and deep eutectic solvents (DES) (Ma et al. 2019) as polymerization components. The latest works are focused mostly on use of DES for green MIP preparation. DES are mixtures of two or more compounds - hydrogen bond donor (HBD; e.g. urea, imidazole derivatives, amides, alcohols, saccharides or organic carboxylic acids) and hydrogen bond acceptor (HBA; quaternary ammonium chlorides e.g. choline chloride) - with a freezing point well below the melting point for any of the original mixture components. In comparison with traditional organic solvents, DESs provide many advantages, such as low toxicity, low volatility, miscibility with water, biocompatibility and biodegradability, low price and they are also easily prepared with a broad scale of polarity. In MIP preparation they can be applied as functional monomers, porogens or modifiers of polymerization mixture what allows due to the presence high amount of functional groups, provide specific interaction with template molecule. The polymers prepared using DESs are characterised by higher selectivity and adsorption capacity, better kinetics, homogenous binding sites, controlled morphology, what make them very perspective for using as stationary phase for HPLC (Viveiros et al. 2018; Roda et al. 2019).

\section{Conclusions}

Molecular imprinting is rapidly developing area of chemistry which have the huge potential in many sectors of applications. MIP are mostly used for chiral separation, since lately is increasing demand for optically pure products. This short review showed different ways of MIPs synthesis for application as stationary phases in HPLC. To achieve efficient separation of enantiomers without band broadening, improvement in MIP preparations and reduction of heterogeneity of binding sites should be done. Significant influence on sorption and separation properties of MIP based CSP have mostly the type of polymerization used in MIP preparation as well as the type of used monomer during imprinting process. On the examples of MIP-amino acids synthesis approaches and results of MIP based chiral HPLC separations, the applicability of these chiral stationary phases were shown. With new, improved strategies for MIP preparation, these sorbents could be suitable for industry for everyday use as highly selective materials for chromatographic separations.

\section{Acknowledgement}

This research was financially supported by Scientific Grant Agency of the Ministry of Education of the Slovak Republic and Slovak Academy of Sciences (grant No. VEGA 1/0159/20).

\section{Conflict of Interest}

The authors declare that they have no conflict of interest. 


\section{References}

Abdollahi E, Abdouss M, Salami-Kalajahi M, Mohammadi A (2016) Molecular recognition ability of molecularly imprinted polymer nano- and micro-particles by reversible addition-fragmentation chain transfer polymerization. Polym. Rev. 56: 557-583.

Alenazi NA, Manthorpe JM, Lai EPC (2015) Selective extraction of BPA in milk analysis by capillary electrophoresis using a chemically modified molecularly imprinted polymer. Food Control. 50: 778-783.

Ansell RJ (2005) Molecularly imprinted polymers for the enantioseparation of chiral drugs. Adv. Drug Deliv. Rev. 57: 1809-1835.

Ansell RJ, Kuah JKL, Wang D, Jackson CE, Bartle KD, Clifford AA (2012) Imprinted polymers for chiral resolution of $( \pm)$-ephedrine, 4: Packed column supercritical fluid chromatography using molecularly imprinted chiral stationary phases. J. Chromatogr. A 1264: 117-123.

Balamurugan K, Gokulakrishnan K, Prakasam T (2012) Preparation and evaluation of molecularly imprinted polymer liquid chromatography column for the separation of Cathine enantiomers. Saudi Pharm. J. 20: 53-61.

Bujak R, Gadzała-Kopciuch R, Nowaczyk A, RaczakGutknecht J, Kordalewska M, Struck-Lewicka W, Waszczuk-Jankowska M, Tomczak E, Kaliszan M, Buszewski B (2016) New sorbent materials for selective extraction of cocaine and benzoylecgonine from human urine samples. J. Pharm. Biomed. Anal. 120: 397-401.

Bystrická Z, Bystrický R, Lehotay J (2016) Thermodynamic study of HPLC enantioseparations of some sulfurcontaining amino acids on teicoplanin columns in ionpairing reversed-phase mode. J. Liq. Chromatogr. Relat. Technol. 39: 775-781.

Cai X, Li J, Zhang Z, Wang G, Song X, You J, Chen L (2014) Chemodosimeter-based fluorescent detection of L-cysteine after extracted by molecularly imprinted polymers. Talanta 120: 297-303.

Chen L, Wang X, Lu W, Wu X, Li J (2016) Molecular imprinting: perspectives and applications. Chem. Soc. Rev. 45: 2137-2211.

Cheong WJ, Song Hee Y, Ali F (2012) Molecular imprinted polymers for separation science: A review of reviews. J. Sep. Sci. 36: 609-628.

Da Silva MS, Vão ER, Temtem M, Mafra L, Caldeira J, Aguiar-Ricardo A, Casimiro T (2010) Clean synthesis of molecular recognition polymeric materials with chiral sensing capability using supercritical fluid technology. Application as HPLC stationary phases. Biosens. Bioelectron. 25: 1742-1747.

Deáková Z, Durăcková Z, Armstrong DW, Lehotay J (2015) Two-dimensional high performance liquid chromatography for determination of homocysteine, methionine and cysteine enantiomers in human serum. J. Chromatogr. A 1408: 118-124.

Fan W, He M, You L, Zhu X, Chen B, Hu B (2016) Watercompatible graphene oxide/molecularly imprinted polymer coated stir bar sorptive extraction of propranolol from urine samples followed by high performance liquid chromatography-ultraviolet detection. J. Chromatogr. A 1443: 1-9.

Fujii N, Takata T, Fujii N, Aki K, Sakaue H (2018) D-Amino acids in protein: The mirror of life as a molecular index of aging. Biochim. Biophys. Acta Proteins Proteom. 1866: 840-847.

Garcia D, Gomez-Caballero A, Guerreiro A, Goicolea MA, Barrio RJ (2015) Molecularly imprinted polymers as a tool for the study of the 4-ethylphenol metabolic pathway in red wines. J. Chromatogr. A 1410: 164-172.

Geibel C, Dittrich K, Woiwode U, Kohout M, Zhang T, Lindner W, Lämmerhofer M (2019) Evaluation of superficially porous particle based zwitterionic chiral ion exchangers against fully porous particle benchmarks for enantioselective ultra-high performance liquid chromatography. J. Chromatogr. A 1603: 130-140.

Giovannoli C, Passini C, di Nardo F, Anfossi L, Baggiani C, Nicholls IA (2018) Affinity capillary electrochromatography of molecularly imprinted thin layers grafted onto silica capillaries using a surface-bound azo-initiator and living polymerization. Polymers 10: 192.

Gutiérrez-Climente R, Gómez-Caballero A, Halhalli M, Sellergren B, Goicolea MA, Barrio RJ (2016) Inifertermediated grafting of molecularly imprinted polymers on porous silica beads for the enantiomeric resolution of drugs. J. Mol. Recognit. 29: 106-114.

Haginaka J, Kagawa C (2004) Chiral resolution of derivatized amino acids using uniformly sized molecularly imprinted polymers in hydro-organic mobile phases. Anal. Bioanal. Chem. 378: 1907-1912.

Hashemi-Moghaddam H, Toosi M, Toosi M (2015) Synthesis and comparison of new layer-coated silica nanoparticles and bulky molecularly imprinted polymers for the solidphase extraction of glycine. Anal. Methods 7: 7488-7495.

Hroboňová K, Deáková Z, Moravčík J, Lehotay J, Armstrong DW, Lomenová A (2015) Separation of methionine enantiomers by using teicoplanin and cyclofructan columns. Nova Biotechnol. Chim. 14: 1-11.

Hroboňová K, Lomenova A, Čižmárik J, Lehotay J (2017) Separation of phenylalanine and methionine enantiomers by HPLC method: A comparison of stationary phase types. Ces. a Slov. Farm. 66: 62-66.

Hroboňová K, Lomenova A (2018) Molecularly imprinted polymer as stationary phase for HPLC separation of phenylalanine enantiomers. Monatsh. Chemie - Chem. Mon. 149: 939-946.

Huang X, Qin F, Chen X, Liu Y, Zou H (2004) Short columns with molecularly imprinted monolithic stationary phases for rapid separation of diastereomers and enantiomers. J. Chromatogr. B 804: 13-18.

Huang X, Zou H, Chen X, Luo Q, Kong L (2003) Molecularly imprinted monolithic stationary phases for liquid chromatographic separation of enantiomers and diastereomers. J. Chromatogr. A 984: 273-282.

Kempe M (1996) Antibody-mimicking polymers as chiral stationary phases in HPLC. Anal. Chem. 68: 1948-1953.

Khan H, Khan T, Park JK (2008) Separation of phenylalanine racemates using d-phenylalanine imprinted microbeads 
as HPLC stationary phase. Sep. Purif. Technol. 62: 363369.

Kibechu RW, Sampath S, Mamba BB, Msagati TAM (2017) Graphene-based molecularly imprinted polymer for separation and pre-concentration of trace polycyclic aromatic hydrocarbons in environmental water samples. J. Appl. Polym. Sci. 134: 45300-45311.

Kim H, Guiochon G (2005) Comparison of the thermodynamic properties of particulate and onolithic columns of molecularly imprinted copolymers. J. Anal. Chem. 77: 93-102.

Kučerová G, Procházková H, Kalíková K, Tesařová E (2016) Sulfobutylether- $\beta$-cyclodextrin as a chiral selector for separation of amino acids and dipeptides in chromatography. J. Chromatogr. A 1467: 356-362.

Kučerová G, Vozka J, Kalíková K, Geryk R, Plecitá D, Pajpanova T, Tesařová E (2013) Enantioselective separation of unusual amino acids by high performance liquid chromatography. Sep. Purif. Technol. 119: 123128.

Li Ji, Hu X, Guan P, Song D, Qian L, Du C, Song R, Wang C (2015) Preparation of "dummy" 1- phenylalanine molecularly imprinted microspheres by using ionic liquid as a template and functional monomer. J. Sep. Sci. 38: 3279-3287.

Li Jie, Lu J, Qiao X, Xu Z (2017) A study on biomimetic immunoassay-capillary electrophoresis method based on molecularly imprinted polymer for determination of trace trichlorfon residue in vegetables. Food Chem. 221: 1285-1290.

Li P, Wang T, Lei F, Peng X, Wang H, Qin L, Jiang J (2017) Preparation and evaluation of paclitaxel-imprinted polymers with a rosin-based crosslinker as the stationary phase in high-performance liquid chromatography. J. Chromatogr. A 1502: 30-37.

Li Q, Yang K, Liang Y, Jiang B, Liu J, Zhang L, Liang Z, Zhang Y (2014) Surface protein imprinted core-shell particles for high selective lysozyme recognition prepared by reversible addition-fragmentation chain transfer strategy. ACS Appl. Mater. Interfaces. 6: 21954-21960.

Liu X-Y, Fang H-X, Yu L-P (2013) Molecularly imprinted photonic polymer based on $\beta$-cyclodextrin for amino acid sensing. Talanta 116: 283-289.

Liyong Z, Guoxiang C, Cong F, Xiaohang L (2003) Tyrosine imprinted polymer beads with different functional monomers via seed swelling and suspension polymerization. Polym. Eng. Sci. 43: 965-974.

Llorina Rañada M, Akbulut M, Abad L, Güven O (2014) Molecularly imprinted poly(N-vinyl imidazole) based polymers grafted onto nonwoven fabrics for recognition/removal of phloretic acid. Radiat. Phys. Chem. 94: 93-97.

Lomenova A, Hroboňová K (2018) Comparison of HPLC Separation of phenylalanine enantiomers on different types of chiral stationary phases. Food Anal. Methods 11: 3314-3323.

Lomenova A, Hroboňová K (2019) Molecularly imprinted polymers as chiral stationary phases in HPLC. Chem. Listy 113: 156-164.
Lucci P, Moret S, Bettin S, Conte L (2017) Selective solidphase extraction using a molecularly imprinted polymer for the analysis of patulin in apple-based foods. J. Sep. Sci. 40: 458-465.

Ma W, An Y, Row KH (2019) Preparation and evaluation of a green solvent-based molecularly imprinted monolithic column for the recognition of proteins by highperformance liquid chromatography. Analyst 144: 63276333.

Maier NM, Lindner W (2007) Chiral recognition applications of molecularly imprinted polymers: A critical review. Anal. Bioanal. Chem. 389: 377-397.

Mergola L, Scorrano S, Del Sole R, Lazzoi MR, Vasapollo G (2013) Developments in the synthesis of a water compatible molecularly imprinted polymer as artificial receptor for detection of 3-nitro-1-tyrosine in neurological diseases. Biosens. Bioelectron. 40: 336-341.

Min Y, Sui Z, Liang Z, Zhang L, Zhang Y (2015) Teicoplanin bonded sub- $2 \mu \mathrm{m}$ superficially porous particles for enantioseparation of native amino acids. J. Pharm. Biomed. Anal. 114: 247-253.

Nakamura Y, Masumoto S, Matsunaga H, Haginaka J (2017) Molecularly imprinted polymer for glutathione by modified precipitation polymerization and its application to determination of glutathione in supplements. J. Pharm. Biomed. Anal. 144: 230-235.

Ndunda EN, Mizaikoff B (2016) Synthesis of stationary phases that provide group recognition for polychlorinated biphenyls by porogenic fragment template imprinting. J. Sep. Sci. 39: 939-946.

Nguy TP, Van Phi T, Tram DTN, Eersels K, Wagner P, Lien TTN (2017) Development of an impedimetric sensor for the label-free detection of the amino acid sarcosine with molecularly imprinted polymer receptors. Sens. Actuators B Chem. 246: 461-470.

Olcer YA, Demirkurt M, Demir MM, Eroglu AE (2017) Development of molecularly imprinted polymers (MIPs) as a solid phase extraction (SPE) sorbent for the determination of ibuprofen in water. RSC Adv. 7: 31441-31447.

Pardeshi S, Singh SK (2016) Precipitation polymerization: A versatile tool for preparing molecularly imprinted polymer beads for chromatography applications. RSC Adv. 6: 23525-23536.

Qin L, He X-W, Zhang W, Li W-Y, Zhang Y-K (2009) Surface-modified polystyrene beads as photografting imprinted polymer matrix for chromatographic separation of proteins. J. Chromatogr. A 1216: 807-814.

Qin L, He X, Li W, Zhang Y (2008) Molecularly imprinted polymer prepared with bonded $\beta$-cyclodextrin and acrylamide on functionalized silica gel for selective recognition of tryptophan in aqueous media. J. Chromatogr. A 1187: 94-102.

Ramstriim O, Nicholls IA, Mosbach K (1994) Synthetic peptide receptor mimics: highly stereoselective recognition in non-covalent molecularly imprinted polymers. Tetrahedron: Asymmetry 5: 649-656.

Reischl RJ, Hartmanova L, Carrozzo M, Huszar M, Frühauf P, Lindner W (2011) Chemoselective and enantioselective 
analysis of proteinogenic amino acids utilizing $\mathrm{N}$-derivatization and 1-D enantioselective anion-exchange chromatography in combination with tandem mass spectrometric detection. J. Chromatogr. A 1218: 83798387.

Riesová M, Geryk R, Kalíková K, Šlechtová T, Voborná M, Martínková M, Bydžovská A, Tesařová E (2016) Direct $\mathrm{CE}$ and HPLC methods for enantioseparation of tryptophan and its unnatural derivatives. Sep. Purif. Technol. 158: 24-30.

Roda A, Matias AA, Paiva A, Duarte ARC (2019) Polymer science and engineering using deep eutectic solvents. Polymers 11: 912.

Sánchez-Hernández L, Bernal JL, Nozal MJ Del, Toribio L (2016) Chiral analysis of aromatic amino acids in food supplements using subcritical fluid chromatography and Chirobiotic T2 column. J. Supercrit. Fluids 107: 519525.

Scorrano S, Mergola L, Sole R Del, Vasapollo G (2011) Synthesis of molecularly imprinted polymers for amino acid derivates by using different functional monomers. Int. J. Mol. Sci. 12: 1735-1743.

Sellergren B, Shea KJ (1993) Influence of polymer morphology on the ability of imprinted network polymers to resolve enantiomers. J. Chromatogr. A 635: 31-49.

Selvolini G, Marrazza G (2017) MIP-based sensors: Promising new tools for cancer biomarker determination. Sensors 17: 718.

Soares M, Vão ER, Temtem M, Mafra L, Caldeira J, Aguiarricardo A, Casimiro $\mathrm{T}$ (2010) Clean synthesis of molecular recognition polymeric materials with chiral sensing capability using supercritical fluid technology. Application as HPLC stationary phases. Biosens. Bioelectron. 25: 1742-1747.

Song YP, Li N, Zhang HC, Wang GN, Liu JX, Liu J, Wang JP (2017) Dummy template molecularly imprinted polymer for solid phase extraction of phenothiazines in meat based on computational simulation. Food Chem. 233: 422-428.

Spivak DA (2005) Optimization, evaluation, and characterization of molecularly imprinted polymers. Adv. Drug Deliv. Rev. 57: 1779-1794.

Sulitzky C, Hall AJ, Lanza F, Unger K (2002) Grafting of molecularly imprinted polymer films on silica supports containing surface-bound free radical initiators. Macromolecules 35: 79-91.

Szultka M, Krzeminski R, Jackowski M, Buszewski B (2013) Simultaneous determination of selected chemotherapeutics in human whole blood by molecularly imprinted polymers coated solid phase microextraction fibers and liquid chromatography-tandem mass spectrometry. J. Chromatogr. B 940: 66-76.

Takeuchi T, Fukuma D, Matsui J (1999) Combinatorial molecular imprinting: An approach to synthetic polymer receptors. Anal. Chem. 71: 285-290.

Takeuchi T, Haginaka J (1999) Separation and sensing based on molecular recognition using molecularly imprinted polymers. J. Chromatogr. B 728: 1-20.

Tamayo FG, Titirici MM, Martin-Esteban A, Sellergren B
(2005) Synthesis and evaluation of new propazineimprinted polymer formats for use as stationary phases in liquid chromatography. Anal. Chim. Acta 542: 38-46.

Tan CJ, Tong YW (2007) Molecularly imprinted beads by surface imprinting. Anal. Bioanal. Chem. 389: 369376.

Tanaka N, Kobayashi H (2003) Monolithic columns for liquid chromatography. Anal. Bioanal. Chem. 376: 298-301.

Tang T, Wei F, Wang X, Ma Y, Song Y, Ma Y, Song Q, Xu G, Cen Y, Hu Q (2018) Determination of semicarbazide in fish by molecularly imprinted stir bar sorptive extraction coupled with high performance liquid chromatography. J. Chromatogr. B 1076: 8-14.

Taujenis L, Olš V, Padarauskas A (2014) Enantioselective determination of protein amino acids in fertilizers by liquid chromatography-tandem mass spectrometry on chiral teicoplanin stationary phase. J. Agric. Food Chem. 62: 11099-11108.

Titirici M, Sellergren B (2006) Thin molecularly imprinted polymer films via reversible addition-fragmentation chain transfer polymerization. Chem. Mater. 18: 1773-1779.

Turiel E, Díaz-Álvarez M, Martín-Esteban A (2016) Supported liquid membrane-protected molecularly imprinted beads for the solid phase micro-extraction of triazines from environmental waters. J. Chromatogr. A 1432: 1-6.

Turiel E, Martin-Esteban A (2004) Molecularly imprinted polymers: towards highly selective stationary phases in liquid chromatography and capillary electrophoresis. Anal. Bioanal. Chem. 378: 1876-1886.

Vasapollo G, Sole RD, Mergola L, Lazzoi MR, Scardino A, Scorrano S, Mele G (2011). Molecularly imprinted polymers: present and future prospective. Int. J. Mol. Sci. 12: 5908-5945.

Vidyasankar S, Ru M, Arnold FH (1997) Molecularly imprinted ligand-exchange adsorbents for the chiral separation of underivatized amino acids. J. Chromatogr. A 775: 51-63.

Viveiros R, Rebocho S, Casimiro T (2018) Green strategies for molecularly imprinted polymer development. Polymers 10: 306-333.

Wagdy HA, Hanafi RS, El-Nashar RM, Aboul-Enein HY (2014) Enantiomeric separation of underivatized amino acids: Predictability of chiral recognition on ristocetin a chiral stationary phase. Chirality 26: 132-135.

Wang H, He Y, He X, Li W, Chen L, Zhang Y (2009) BSAimprinted synthetic receptor for reversible template recognition. J. Sep. Sci. 32: 1981-1986.

Wei X, Husson SM (2007) Surface-grafted, molecularly imprinted polymers grown from silica gel for chromatographic separations. Ind. Eng. Chem. Res. 46: 2117-2124.

Woiwode U, Neubauer S, Lindner W, Buckenmaier S, Lämmerhofer M (2018) Enantioselective multiple heartcut two-dimensional ultra-high-performance liquid chromatography method with a Coreshell chiral stationary phase in the second dimension for analysis of all proteinogenic amino acids in a single run. J. Chromatogr. A 1562: 69-77. 
Xiao H, Peng J, Peng H, Bu L, Pan Z, He Y, Chen Y, Chen F, Gong C, Tang Q (2016) Preparation and evaluation of surface molecularly imprinted polymers as stationary phase columns for high performance liquid chromatography. Anal. Methods 8: 7951-7958.

Yan H, Row KH (2006) Characteristic and synthetic approach of molecularly imprinted polymer. Int. J. Mol. Sci. 7: 155178.

Yang Y-J, Liu X-W, Kong X-J, Qin Z, Jiao Z-H, Li S-H, Li J$Y$ (2018) Preparation and evaluation of oseltamivir molecularly imprinted polymer silica gel as liquid chromatography stationary phase. Molecules 23: 18811895.

Yilmaz E, Ramström O, Möller P, Sanchez D, Mosbach K (2002) A facile method for preparing molecularly imprinted polymer spheres using spherical silica templates. J. Mater. Chem. 12: 1577-1581.

Yoshimatsu K, Reimhult K, Krozer A, Mosbach K, Sode K, Ye L (2007) Uniform molecularly imprinted microspheres and nanoparticles prepared by precipitation polymerization: The control of particle size suitable for different analytical applications. Anal. Chim. Acta 584: 112-121.

Yu C, Mosbach K (2000) Influence of mobile phase composition and cross-linking density on the enantiomeric recognition properties of molecularly imprinted polymers. J. Chromatogr. A 888: 63-72.

Zhang Z, Zhang M, Liu Y, Yang X, Luo L, Yao S (2012) Preparation of $\mathrm{L}$-phenylalanine imprinted polymer based on monodisperse hybrid silica microsphere and its application on chiral separation of phenylalanine racemates as HPLC stationary phase. Sep. Purif. Technol. 87: 142-148.

Zheng C, Huang Y-P, Liu Z (2011) Recent developments and applications of molecularly imprinted monolithic column for HPLC and CEC. J. Sep. Sci. 34: 1988-2002.

Zhu F, Wang J, Zhu L, Tan L, Feng G, Liu S, Dai Y, Wang H (2016) Preparation of molecularly imprinted polymers using theanine as dummy template and its application as SPE sorbent for the determination of eighteen amino acids in tobacco. Talanta 150: 388-398. 